\title{
Factores determinantes de la satisfacción laboral en España antes y durante la crisis de 2008
}

\author{
María Carmen Sánchez-Sellero ${ }^{1}$ (D), Pedro Sánchez-Sellero² ${ }^{[D}$ \\ ${ }^{1}$ Universidade da Coruña (Spain) \\ ${ }^{2}$ Universidad de Zaragoza (Spain) \\ c.sanchez@udc.es,pedross@unizar.es
}

Received July, 2016

Accepted August, 2016

\section{Resumen}

Objeto: Obtendremos diferencias entre características inherentes al trabajo y de tipo personal, con el fin de valorar cuáles explican mejor la satisfacción laboral. El estudio se realiza en un año de crecimiento y dos de crisis económica con el fin de averiguar si la crisis altera los resultados anteriores.

Diseño/metodología/enfoque: Los datos proceden de la Encuesta de calidad de vida en el trabajo (Ministerio de Empleo y Seguridad Social), para los años 2007, 2009 y 2010. Emplearemos modelos lineales (ANOVA), análisis de componentes principales y regresión múltiple stepwise. Las variables son Grado de satisfacción con el trabajo actual y una combinación de variables personales (sexo, edad, nivel de estudios) e inherentes al empleo (hasta 14 variables dependiendo del método utilizado).

Aportaciones y resultados: Mediante modelos lineales obtenemos que las variables referidas al trabajo proporcionan mejores resultados para explicar la satisfacción laboral, y tras una regresión stepwise elaborada a partir de los factores de un análisis de componentes principales comprobamos que el salario es uno de los últimos factores en esta explicación. Las variables que 
influyen en la satisfacción laboral no dependen del ciclo económico, aunque las jerarquías entre ellas son distintas.

Implicaciones sociales: Durante la crisis, las exigencias de los trabajadores disminuyen ya que es preferible tener un empleo en inferiores condiciones y menos remunerado que, por el contrario, no tenerlo. La reducción del grado de satisfacción con la estabilidad y con el salario es fruto del panorama económico, pues los contratos laborales son menos estables y los salarios menores.

Originalidad / Valor añadido: Comparamos los resultados obtenidos de la regresión stepwise realizada con las variables originales y con los factores de los componentes principales. La combinación de estas metodologías es novedosa en los estudios de satisfacción laboral, así como la original combinación de las 14 variables relativas al trabajo.

Palabras clave: Satisfacción laboral, Motivación, Remuneración, Género, Crisis económica

Códigos JEL: J21, J22, J28, J30, J81, C13, C25

Title: Determinants of job satisfaction in Spain before and during the economic crisis of 2008

\section{Abstract}

Purpose: We try to find out differences between personal and job-related features to know which better explain job satisfaction. This study is made in a year of economic growth and in two years of economic crisis, in order to determine if the economic crisis affects to previous results.

Design/methodology: The data are from the Quality of Labour Life Survey by the Ministry of Employment and Social Security in Spain, in 2007, 2009 and 2010. We use linear models (ANOVA), principal component analysis and stepwise multiple regression. The variables are degree of satisfaction with the current job and a group of personal variables (gender, age and education level) and job-related variables (with a maximum of 14 variables depending on the method).

Findings: Using linear models get the variables related to work which provide better results to explain job satisfaction, and after a stepwise regression made with factors of principal component analysis, we find out that salary is one of the last factors in this explanation. The 
variables that influence on job satisfaction do not depend on the economic cycle, although the hierarchies are different among them.

Social implications: During the crisis, the demands of workers are lower because they prefer to have a job with low working conditions and low salary than lose their job. Reducing the degree of satisfaction with stability and wages is due to the economic situation, because labour contracts are less stable and remunerated.

Originality/value: We have compared the results of stepwise regression made with the original variables and the factors of principal component analysis. The combination of these methodologies is new in studies of job satisfaction, as well as the original combination of 14 variables related to work.

Keywords: Job satisfaction, Motivation, Remuneration, Gender, Economic crisis

Jel Codes: J21, J22, J28, J30, J81, C13, C25

\section{Introducción}

Los factores que pueden influir en la satisfacción laboral son muchos, y muchos son también los autores que desde distintas vertientes han analizado el grado de afectación de esos factores sobre la satisfacción de los trabajadores. En este artículo utilizaremos los microdatos de la Encuesta de calidad de vida en el trabajo (ECVT), facilitados por el Ministerio de Empleo y Seguridad Social correspondientes a los año 2007, 2009 y 2010 (el último año disponible en 2016 es 2010), con la finalidad de explicar el grado de satisfacción con el trabajo actual a partir de varias variables independientes (personales e inherentes al trabajo). Se van probando distintos modelos que dividimos en tres grupos: en un primer grupo se incluyen sólo las características personales, en un segundo grupo características de la organización, y en un tercer grupo, ambas características. El interés reside en conocer cuál de estos tipos de variables explica mejor la satisfacción laboral. Para ello, se utiliza la modelización lineal general (ANOVA). Una vez cumplido este objetivo, y mediante un análisis de componentes principales (ACP) tratamos de eliminar la interacción de 14 variables explicativas -todas ellas referidas al trabajo; con los factores obtenidos aplicamos una regresión lineal múltiple stepwise, con la que deseamos conocer cuáles son las que ejercen mayor influencia en la variable dependiente 
(estableciendo una jerarquía entre ellas). Por último, comparamos los resultados obtenidos de la regresión stepwise con las variables originales (sin hacer componentes principales) y la regresión stepwise hecha con los factores de los componentes principales. La combinación de estas metodologías es totalmente novedosa en los estudios de satisfacción laboral, en concreto, la utilización de los factores extraídos de un análisis de componentes principales como variables explicativas en una regresión stepwise; además, se aplican en años correspondientes a distinto ciclo económico.

Este artículo tiene la particularidad de ser analizado en el contexto del mercado laboral español afectado por una de las fases de crisis económica más larga. Al repetir el proceso en 2007 (año de crecimiento en la economía española) y 2009 y 2010 (crisis económica), trataremos de averiguar si la crisis económica que se inicia en 2008 hace variar los factores que ejercen mayor influencia en la satisfacción laboral.

Establecemos cuatro objetivos de partida. El primero es averiguar si las variables relativas al trabajo son las que ejercen mayor influencia en la satisfacción laboral. Si se confirma este supuesto, el segundo objetivo consistirá en englobar estas variables en grupos homogéneos. En tercer lugar tratamos de conocer la influencia del cambio de ciclo económico en las variables de satisfacción. Y el cuarto es establecer una jerarquía para saber cuáles son las variables independientes y/o factores obtenidos en el análisis ACP que ejercen mayor influencia en la satisfacción laboral.

Partimos del supuesto de que los determinantes de la satisfacción laboral son básicamente los mismos en España que en los demás países europeos y occidentales. Por ello, en la revisión teórica incluimos los estudios de autores correspondientes a distintos países.

En el epígrafe 2 de este trabajo se repasa el concepto de satisfacción laboral, así como las variables o factores determinantes del mismo. El epígrafe 3 se dedica a los datos de partida, en este caso, la ECVT (años 2007, 2009 y 2010). El epígrafe 4 (resultados) expone la metodología utilizada: modelo lineal general (ANOVA), análisis de componentes principales, y regresión lineal múltiple stepwise. Utilizando estas técnicas se van desarrollando distintos modelos que difieren básicamente en el tipo de variables incluidas, se añaden los comentarios pertinentes a los resultados obtenidos y la idoneidad del modelo (epígrafe 5). Y por último, el epígrafe 6 se destina a las conclusiones del trabajo. 


\section{Revisión teórica}

El concepto de satisfacción laboral ha sido y es ampliamente tratado. Sus inicios se deben a Hoppock, que en 1935 abarcando distintos grupos de población, desarrolló los primeros estudios sobre este tema. Zhu (2013) revisa más de una década de investigaciones sobre los antecedentes y resultados de satisfacción en el trabajo. Otro repaso teórico lo podemos encontrar en Aziri (2011). La satisfacción laboral incide en la actitud del trabajador en el desarrollo de sus obligaciones laborales. La idea de satisfacción surge de la comparación entre el trabajo real y las expectativas que el trabajador se había generado. El trabajador estará insatisfecho si cree que está en desventaja respecto a sus compañeros, del mismo modo que si considera que el trabajo anterior le ofrecía mejores condiciones. A mayor satisfacción laboral, mayor compromiso del trabajador en el desempeño de sus tareas; por el contrario, si la satisfacción en el trabajo es escasa o nula, no pondrá demasiado empeño en su actividad diaria.

\subsection{Influencia de las características personales en la satisfacción laboral}

En las investigaciones de Koustelios (2001) se examinó la relación entre las características personales y aspectos específicos de la satisfacción laboral. Okpara (2004, 2006) demuestra que las características personales como el sexo, edad, etc. son predictoras significativas de la satisfacción en el trabajo. Bowling (2010) se refiere a algunos estudios de comportamiento que han examinado las interacciones entre la personalidad y las actitudes laborales. Franek y Vecera (2008) examinan la relación entre la satisfacción laboral y una selección de variables personales en un grupo de personas pertenecientes a distintas áreas ocupacionales.

En el colectivo estudiado por Ghazzawi (2011) se aceptaron las hipótesis nulas de que la edad no juega un papel en la satisfacción laboral. En el trabajo de Clark, Oswald y Warr (2011) se expone que, en líneas generales, la satisfacción laboral aumenta linealmente con la edad; sin embargo, hay argumentos y evidencias empíricas que establecen que la relación tiene forma de $\mathrm{U}$, disminuyendo moderadamente en los primeros años de empleo y aumentando constantemente hasta su jubilación. Kooij, Jansen, Dikkers y De Lange (2010) estudian la influencia de la edad sobre las asociaciones entre las prácticas de recursos humanos y la satisfacción en el trabajo. Boumans, De Jong y Janssen (2011) investigaron la influencia de la edad sobre la relación entre las características del trabajo, la motivación en el trabajo y la satisfacción laboral. 
Donohue y Heywood (2004) analizan la satisfacción laboral en función del género. Westover (2012a) establece que del mismo modo que la satisfacción laboral afecta a una gran variedad de resultados sociales, las organizaciones deben reconocer las diferencias de género en este concepto. En muchas investigaciones se constata que las mujeres presentan un nivel superior de satisfacción laboral al de los varones. El trabajo de Sánchez, Fuentes y Artacho (2007) avala también este resultado. Se revisan las diferencias entre hombres y mujeres respecto a la influencia de otras variables sociodemográficas, del puesto y de la organización sobre la satisfacción con el trabajo.

Hay autores que encuentran relación entre la edad y el género como Bellou (2010); este autor establece que el género de los empleados y la edad influyen en la forma en que los valores de la organización afectan a la satisfacción en el trabajo. Carrillo-García, Solano-Ruíz, Martínez-Roche y Gómez-García (2013) delimitan la influencia del género y la edad en los niveles de satisfacción laboral, obteniendo asociaciones significativas entre ambas variables; respecto al género, las mujeres se muestran más satisfechas y en cuanto a la edad son los profesionales de entre 20-30 años y los mayores de 61 los que manifiestan mayor satisfacción. En Gazioglu y Tansel (2006), se confirma la teoría de que las mujeres están más satisfechas que los hombres, y una relación en forma de U entre satisfacción y edad.

Según Allen y Van der Velden (2001), la educación repercute sobre los salarios y otros resultados del mercado laboral; para estos autores, las distintas habilidades son mucho mejores predictores de la satisfacción en el trabajo que el nivel educativo. En la influencia de las características personales (edad, sexo, estudios) sobre la satisfacción laboral, Gamero (2005) destaca el papel que juegan las expectativas laborales de las personas; cuanto menor sean las expectativas del trabajador, más probable será que se considere satisfecho. En este sentido, Linz y Semykina (2013) relacionan satisfacción laboral, expectativas y género en varios países.

\subsection{Influencia de las características propias del trabajo y del entorno en la satisfacción laboral}

En Judge, Thoresen, Bono y Patton (2001) se realiza una revisión cualitativa y cuantitativa de la relación entre la satisfacción laboral y el desempeño laboral. Chiang, Martín y Núñez (2010) afirman que el concepto de satisfacción laboral es esencial en el análisis del comportamiento organizacional porque para la mayoría de los trabajadores representa o bien un fin en sí mismo, o bien un medio para conseguir la satisfacción personal. La satisfacción laboral se asocia a indicadores organizacionales como la calidad del desempeño, el rendimiento, etc. 
Bòria-Reverter, Crespi-Vallbona y Mascarilla-Miró (2012) analizan los posibles nexos de unión entre salario, distintos activos intangibles y la satisfacción laboral en las organizaciones. Incorporan el concepto de clima organizacional, basado en atributos del entorno laboral. Si los trabajadores perciben un buen clima organizacional, sus comportamientos serán más adecuados para el bienestar de la empresa. Para Cuadra-Peralta y Veloso-Besio (2010), los cimientos de un buen clima laboral se relacionan básicamente con el adecuado funcionamiento de la organización, y más específicamente con los siguientes indicadores: conciliación del trabajo con la vida familiar, prestaciones de tipo social, satisfacción en el puesto de trabajo y calidad directiva (liderazgo), entre otros. Robles-García, DierssenSotos, Martínez-Ochoa, Herrera-Carral, Díaz-Mendi y Llorca-Díaz (2005) hablan de clima organizacional; para ellos, la satisfacción está fuertemente asociada a la valoración positiva de características propias de la organización.

La motivación laboral son los estímulos que una persona posee que lo conducen a actuar de peor o mejor manera en el ámbito laboral. Pueden proceder del trabajo o de su círculo personal (familia, amigos). Pérez (1997), desde una perspectiva histórico-sociológica, estudia diferentes planteamientos en torno a la motivación y la satisfacción laboral partiendo de las aspiraciones y expectativas del trabajador. En el estudio de Danish y Usman (2010) se dice que los recursos humanos son lo más importante entre todos los recursos que una organización posee; conservar personal eficiente y experimentado es crucial en el rendimiento de una organización. Este estudio intenta encontrar los factores más importantes que motivan a los empleados; los empleados motivados pueden hacer una organización más competitiva, con más valor y rentable. Los resultados de la investigación de Alnıaçı, Alnıaçı, Akçin y Erat (2012) mostraron que la motivación tiene una correlación positiva con el compromiso organizacional y la satisfacción laboral. Las características individuales, excepto el género de los encuestados no ejercieron ninguna asociación significativa con la motivación.

En la teoría de la motivación de Lawler y Porter (1967), la relación entre la expectativa y la recompensa obtenida produce la satisfacción o la insatisfacción laboral. Por ello, si la recompensa obtenida por el rendimiento en el trabajo excede de la que considera adecuada o si es equiparable, el trabajador alcanza el estado de satisfacción. Si esta relación se produce en sentido inverso, se produce la insatisfacción. En esta teoría, el término recompensa no solo se refiere a remuneración económica, sino que incluye un amplio abanico de resultados (sea reconocimientos, ascensos, valoración de superiores, etc.). Linz y Semykina (2012) obtuvieron una fuerte relación positiva entre la satisfacción laboral y las variables de recompensa, incluso si la recompensa esperada no es muy deseada. Mientras que los ingresos propios 
normalmente están positivamente relacionados con la satisfacción laboral, los ingresos de los compañeros pueden ser positiva o negativamente vinculados a la satisfacción laboral.

Petrescu y Simmons (2008) investigan la relación entre la gestión de recursos humanos y la satisfacción de los trabajadores con su remuneración. Centran su estudio en el impacto sobre la satisfacción laboral general y la satisfacción con la remuneración. Hofmans, De Gieter y Pepermans (2013) estudian las diferencias individuales en la relación entre la satisfacción con la remuneración y la satisfacción laboral. Singh y Loncar (2010) examinan las relaciones entre satisfacción con el salario, satisfacción con el trabajo y cambio de empleo. Card, Mas, Moretti y Saez (2012) sugieren que la satisfacción en el trabajo depende directamente de las comparaciones salariales con los compañeros y que esta relación no es lineal. En Judge, Piccolo, Podsakoff, Shaw y Rich (2010) se obtiene una correlación de 0,15 entre el nivel salarial y la satisfacción laboral. Los resultados del estudio de Tremblay, Chênevert y Hébert (2012) demostraron que las motivaciones familiares y aquellas vinculadas al desarrollo profesional producen un efecto positivo sobre la satisfacción; sólo las buenas condiciones económicas no son suficientes. Böckerman, Ilmakunnas y Johansson (2011) concluyeron que incrementos en los salarios no implicaban incrementos en la satisfacción.

En Gamero (2005) se afirma que la estabilidad laboral y las posibilidades de promoción tienen un fuerte impacto sobre la satisfacción en el trabajo. En el estudio de los factores que explican la satisfacción de los trabajadores asalariados en España, las características laborales con mayor efecto marginal sobre la probabilidad de estar muy satisfecho con el empleo actual son, por este orden, la tarea, la estabilidad del empleo, la dificultad para conciliar el ámbito laboral con el familiar, la percepción sobre la recompensa económica, y las relaciones con los jefes inmediatos. También repercute de manera considerable el entorno físico en el que se desarrolla la tarea y el desajuste con respecto a la carga de trabajo.

En un ámbito más extenso, citamos el trabajo de Gazioglu y Tansel (2006), en el que cuatro medidas diferentes de satisfacción en el trabajo están relacionadas con una variedad de características personales y laborales. Para Cohrs, Abele y Dette (2006), la satisfacción en el trabajo puede ser conceptualizada como una función de las condiciones situacionales, las características personales y las interacciones entre ambos grupos de variables.

El propósito de Westover y Taylor (2010) es explorar las diferencias entre distintos países en las satisfacciones del trabajo y sus determinantes en el tiempo. En un mercado global cada vez más competitivo, las organizaciones se preguntan cómo sacar más provecho de sus empleados. Las distintas 
soluciones pasan por la difusión y el fortalecimiento de los valores fundamentales de la organización, y la creencia de que un aumento de la satisfacción laboral produce beneficios en la productividad, y ayuda a crear un compromiso de alto rendimiento. Para Westover (2012b), la satisfacción laboral es un concepto dinámico que cambia en respuesta a las condiciones personales y ambientales. Es a través del tiempo y en diferentes contextos como se examinan y comprenden mejor los factores principales que afectan a la satisfacción laboral.

En esta revisión teórica hemos querido hacer un repaso de los factores que pueden influir en la satisfacción laboral, haciendo especial hincapié en aquellos que vamos incluir en nuestro análisis empírico posterior. Como factores personales incluiremos la variable edad, el género y el nivel de estudios y como factores propios del trabajo, la motivación, promociones, salario, entre otras variables. Repetiremos el estudio en distintos años para averiguar si el comportamiento difiere durante la crisis económica que se inicia en 2008.

\section{Datos, métodos y variables}

Los análisis que se exponen en este estudio se basan en los microdatos de la Encuesta de calidad de vida en el trabajo (ECVT) facilitados por el Ministerio de Empleo y Seguridad Social y correspondientes a los años 2007, 2009 y 2010. El ámbito geográfico de esta encuesta es todo el territorio español, exceptuando a Ceuta y Melilla. El ámbito poblacional está delimitado por la población ocupada de 16 y más años que reside en viviendas familiares. El cuestionario de la ECVT está formado por distintos bloques, debido a la existencia de múltiples objetivos en la investigación. Estos bloques son:

- Datos sociodemográficos: se recoge la edad, el sexo y el nivel de estudios.

- Situación laboral. Ocupación: engloba variables que describen el puesto de trabajo.

- Calidad de vida en el trabajo: satisfacción en el trabajo, organización del trabajo, entorno laboral, relaciones laborales, tiempo de trabajo, seguridad en el trabajo, formación académica y profesional, compensación por el trabajo, conciliación de la vida laboral y la vida familiar, actitudes y opiniones, negociación colectiva, asociacionismo, y movilidad laboral y geográfica.

La muestra de la ECVT se diseñó mediante un muestreo trietápico estratificado. Como estratos se han considerado el cruce de comunidad autónoma y tramo de tamaño de municipio. En cada estrato se 
seleccionaron, en la primera etapa, una muestra de secciones censales con probabilidades proporcionales al tamaño de cada sección; en la segunda etapa, se seleccionó, de cada sección censal elegida en la primera, una muestra de viviendas con probabilidad igual para cada una de las viviendas de la sección; por último, y dentro de cada vivienda seleccionada en la segunda etapa, se eligió aleatoriamente una persona ocupada residente en la misma.

En esta investigación, el ámbito de aplicación es todo el colectivo de trabajadores en España. La encuesta ECVT la forman 7782, 7981 y 8061 personas en los años 2007, 2009 y 2010, pero al eliminar la falta de respuesta en algunas preguntas, queda en 5656, 6274 y 5841 personas (al final). Para extender los resultados a la población, se ha de ponderar por los factores de elevación correspondientes; representan en torno a 18-20 millones de personas. La metodología utilizada, tanto la relativa a los modelos lineales generales (ANOVA), como el análisis de componentes principales y la regresión lineal múltiple stepwise, se justifica no solo por el tipo de variables implicadas sino también por la propia finalidad del estudio. A lo largo de la exposición de estos modelos también se irán comprobando algunos resultados que confirman la idoneidad de los métodos aplicados.

Nuestro primer objetivo pretende dar contestación a la pregunta ¿Son las variables relativas al trabajo las que ejercen mayor influencia en la satisfacción laboral? Para ello,comenzamos definiendo la variable dependiente que es el grado de satisfacción con el trabajo actual (GStrabajoactual), y las variables independientes que son: sexo, edad (categórica), nivel de estudios (todas estas, variables cualitativas), y grado satisfacción-motivación (GSmotivación), grado satisfacción actividad (GSactividad), grado satisfacción organización trabajo (GSorg.trabajo) y grado satisfacción salario (GSsalario) (todas ellas cuantitativas, toman valores de 0 a 10). En 2007 sustituimos la variable GSmotivación por GSambiente.trabajo porque la primera no está en la base de datos en ese año.

Reconocemos que por características laborales se entiende, por ejemplo, el tipo de jornada, el salario, el sector de actividad, etc. El grado de satisfacción con el salario (por ejemplo) es una característica personal ya que depende de cada persona, pues es posible que dos personas que cobren el mismo salario tengan distinto nivel de satisfacción con la remuneración que perciben. Tampoco son características personales "al uso" como pueden ser la edad o el sexo. Se podrían entender como características pseudo-laborales ya que se refieren a una percepción personal de una característica laboral como es el salario, la actividad, etc.

En la Tabla 1 figuran los modelos iniciales que vamos a desarrollar (modelos lineales generales, ANOVA), y en la Tabla 2 la descripción de las variables. En los modelos del grupo 1 las variables 
independientes son las características personales, en el modelo del grupo 2 figuran las características propias del trabajo (pseudo-laborales), y en los modelos del grupo 3 incluimos ambos tipos de variables. En el trabajo de Spagnoli, Caetano y Santos (2012) se hace también un estudio de satisfacción laboral utilizando modelos ANOVA en distintos años.

\begin{tabular}{|c|c|c|c|c|}
\hline \multirow{2}{*}{ Modelos } & \multirow{2}{*}{ Variables independientes } & Año 2007 & Año 2009 & Año 2010 \\
\hline & & $\mathbf{R}^{2}$ & $\mathbf{R}^{2}$ & $\mathbf{R}^{2}$ \\
\hline & Sexo & $0.021^{\mathrm{a}}$ & $0.007^{\mathrm{a}}$ & $0.015^{\mathrm{a}}$ \\
\hline Modelos grupo 1 & $\begin{array}{l}\text { Edad (categórica) } \\
\text { Nivel de estudios }\end{array}$ & $0.036^{\mathrm{b}}$ & $0.021^{b}$ & $0.035^{\mathrm{b}}$ \\
\hline Modelo grupo 2 & $\begin{array}{l}\text { GSmotivación* } \\
\text { GSactividad } \\
\text { GSorganizacióntrabajo } \\
\text { GSsalario }\end{array}$ & 0.633 & 0.478 & 0.572 \\
\hline Modelos grupo 3 & $\begin{array}{l}\text { Sexo } \\
\text { Edad (categórica) } \\
\text { Nivel de estudios } \\
\text { GSmotivación* } \\
\text { GSactividad } \\
\text { GSorganizacióntrabajo } \\
\text { GSsalario }\end{array}$ & $\begin{array}{l}0.635^{\mathrm{c}} \\
0.637^{\mathrm{d}} \\
0.641^{\mathrm{e}}\end{array}$ & $\begin{array}{l}0.481^{\mathrm{c}} \\
0.483^{\mathrm{d}} \\
0.489^{\mathrm{e}}\end{array}$ & $\begin{array}{l}0.575^{\mathrm{c}} \\
0.578^{\mathrm{d}} \\
0.587^{\mathrm{e}}\end{array}$ \\
\hline
\end{tabular}

Nota: a. modelo personalizado (con efectos principales e interacciones del sexo con las demás variables cualitativas). b. modelo factorial (con todas las interacciones). c. modelo de efectos principales. d. modelo personalizado (con efectos principales e interacciones del sexo con las demás variables cualitativas). e. modelo factorial (con todas las interacciones).

* La variable GSmotivación no existe en el año 2007, por eso en ese año hemos utilizado la variable GSambiente.trabajo.

Variable dependiente: GStrabajoactual.

Tabla 1. Grupos de modelos lineales y variables incluidas, por años Fuente: elaboración propia a partir de ECVT (Ministerio de Empleo y Seguridad Social: 2007, 2009 y 2010) 


\begin{tabular}{|c|c|}
\hline Variables independientes & Categorías o valores \\
\hline Sexo & $\begin{array}{l}\text { 1. Varón } \\
\text { 2. Mujer }\end{array}$ \\
\hline Edad (categórica) & $\begin{array}{l}\text { 1. 16-25 años } \\
\text { 2. 26-35 años } \\
\text { 3. 36-45 años } \\
\text { 4. 46-55 años } \\
\text { 5. } 56-65 \text { años, } \\
\text { 6. más de } 65 \text { años }\end{array}$ \\
\hline Nivel de estudios & $\begin{array}{l}\text { 1. No sabe leer ni escribir } \\
\text { 2. Menos que estudios primarios } \\
\text { 3. Enseñanza primaria } \\
\text { 4. Enseñanza secundaria } \\
\text { 5. Ciclos formativos de grado medio (Formación profesional FPI) } \\
\text { 6. Ciclos formativos de grado superior (Formación profesional FPII) } \\
\text { 7. Bachiller superior, BUP, COU o equivalentes } \\
\text { 8. Estudios universitarios de grado medio } \\
\text { 9. Estudios universitarios de grado superior de segundo o tercer ciclo }\end{array}$ \\
\hline GSmotivación* & De 0 a10 \\
\hline GSactividad & De 0 a10 \\
\hline GSorganizacióntrabajo & De 0 a10 \\
\hline GSsalario & De 0 a10 \\
\hline
\end{tabular}

Tabla 2. Variables utilizadas en los modelos y sus categorías o valores. Fuente: elaboración propia a partir de ECVT (Ministerio de Empleo y Seguridad Social: 2007, 2009 y 2010)

\section{Resultados}

En la Tabla 1 se pone de manifiesto que los modelos del grupo 1 no sirven para explicar la variación en la satisfacción laboral de los trabajadores (1.5\% y 3.5\% es el porcentaje explicado en 2010, dependiendo de si el modelo es de efectos principales con todas o algunas interacciones); escaso nivel de explicación de las variables personales se muestra también en el trabajo de Dueñas, Iglesias y Llorente (2010). Mientras las características personales (modelos grupo 1) explican entre 1-4\% aproximadamente de la satisfacción laboral, las características propias del trabajo (modelo grupo 2) explican más de un 50\% en 2007 y 2010.

En parecidos términos, los resultados del estudio de Mpeka (2012) demostraron que los compañeros de trabajo, remuneración, promoción, supervisión y trabajo en sí, tienen una influencia significativa en la satisfacción laboral, mientras que la edad y el género no influyen. Por lo tanto, se deben promover activamente esos factores en el lugar de trabajo con el fin de fomentar un mejor desempeño en el trabajo. 
De los modelos del grupo 3, en el que intervienen características personales y propias del trabajo, desarrollamos en la Tabla 3 el modelo con efectos principales, $\mathrm{R}^{2}=0.575$ en 2010. Elegimos este modelo, aunque poca diferencia hay en la bondad del modelo con los demás de ese grupo, porque en el modelo factorial las salidas informáticas de resultados son demasiado extensas. En la Tabla 3 también aparecen las estimaciones del grado en que cada factor está afectando a la variable dependiente. El estadístico eta cuadrado parcial, que para un efecto $\mathrm{A}$ se obtiene de la siguiente manera: $\left(\mathrm{F}_{\mathrm{A}}{ }^{*} \mathrm{gl}_{\mathrm{A}}\right) /\left(\mathrm{F}_{\mathrm{A}}{ }^{*}\right.$ $\mathrm{gl}_{\mathrm{A}}+\mathrm{gl}_{\text {error }}$ ) siendo $\mathrm{F}_{\mathrm{A}}$ el estadístico de $\mathrm{A}$ y gl los grados de libertad, se interpreta como la proporción de la varianza explicada, es decir, es una estimación de la proporción de la variación de la variable dependiente que está explicada por cada efecto. En este caso, se observa que los valores más altos de este estadístico corresponden a las variables GSmotivación o GSambiente.trabajo, GSactividad, GSorg.trabajo y GSsalario, con lo cual, son los factores que afectan en mayor grado a la explicación de la satisfacción laboral (GStrabajoactual). De igual modo, Sánchez-Sellero Sánchez-Sellero, CruzGonzález y Sánchez-Sellero (2016) aplican modelización lineal en un estudio de satisfacción laboral en España en este período de crisis.

\begin{tabular}{|c|c|c|c|c|c|c|}
\hline \multicolumn{7}{|c|}{ AÑO 2007} \\
\hline Fuente & $\begin{array}{c}\text { Suma de } \\
\text { cuadrados tipo III }\end{array}$ & gl & Media cuadrática & $\mathbf{F}$ & Sig. & $\begin{array}{c}\text { Eta cuadrado } \\
\text { parcial }\end{array}$ \\
\hline Modelo corregido & $33842162.856^{\mathrm{a}}$ & 19 & 1781166.466 & 1647966.346 & 0.000 & 0.635 \\
\hline Intersección & 513940.150 & 1 & 513940.150 & 475506.409 & 0.000 & 0.026 \\
\hline Sexo & 3145.289 & 1 & 3145.289 & 2910.077 & 0.000 & 0.000 \\
\hline Edad (categórica) & 27493.045 & 5 & 5498.609 & 5087.409 & 0.000 & 0.001 \\
\hline Nivel de estudios & 63365.347 & 9 & 7040.594 & 6514.081 & 0.000 & 0.003 \\
\hline GSambientetrabajo & 878831.380 & 1 & 878831.380 & 813110.153 & 0.000 & 0.043 \\
\hline GSactividad & 3326592.193 & 1 & 3326592.193 & 3077821.240 & 0.000 & 0.146 \\
\hline GSorg.trabajo & 3018320.248 & 1 & 3018320.248 & 2792602.648 & 0.000 & 0.134 \\
\hline GSsalario & 4074243.667 & 1 & 4074243.667 & 3769561.450 & 0.000 & 0.173 \\
\hline Error & 19470357.431 & 18014315 & 1.081 & & & \\
\hline Total & 987074979.000 & 18014335 & & & & \\
\hline Total corregida & 53312520.287 & 18014334 & & & & \\
\hline \multicolumn{7}{|c|}{ AÑO 2009} \\
\hline Fuente & $\begin{array}{c}\text { Suma de } \\
\text { cuadrados tipo III }\end{array}$ & gl & Media cuadrática & $\mathbf{F}$ & Sig. & $\begin{array}{c}\text { Eta cuadrado } \\
\text { parcial }\end{array}$ \\
\hline Modelo corregido & $32189557.703^{\mathrm{a}}$ & 18 & 1788308.761 & 966824.414 & 0.000 & 0.481 \\
\hline Intersección & 1237235.292 & 1 & 1237235.292 & 668894.160 & 0.000 & 0.034 \\
\hline Sexo & 59416.460 & 1 & 59416.460 & 32122.688 & 0.000 & 0.002 \\
\hline Edad (categórica) & 34139.946 & 5 & 6827.989 & 3691.458 & 0.000 & 0.001 \\
\hline Nivel de estudios & 131399.672 & 8 & 16424.959 & 8879.927 & 0.000 & 0.004 \\
\hline GSmotivación & 2390400.055 & 1 & 2390400.055 & 1292336.750 & 0.000 & 0.064 \\
\hline GSactividad & 3516135.741 & 1 & 3516135.741 & 1900950.189 & 0.000 & 0.092 \\
\hline GSorg.trabajo & 1106867.267 & 1 & 1106867.267 & 598412.489 & 0.000 & 0.031 \\
\hline GSsalario & 1863629.311 & 1 & 1863629.311 & 1007545.428 & 0.000 & 0.051 \\
\hline Error & 34749117.350 & 18786630 & 1.850 & & & \\
\hline Total & 1075186923.000 & 18786649 & & & & \\
\hline Total corregida & 66938675.053 & 18786648 & & & & \\
\hline
\end{tabular}




\begin{tabular}{|l|r|r|r|r|r|r|}
\hline \multicolumn{1}{|c|}{ Fuente } & \multicolumn{1}{c|}{$\begin{array}{c}\text { Suma de } \\
\text { cuadrados tipo III }\end{array}$} & g1 & Media cuadrática & \multicolumn{1}{c|}{ F } & Sig. & $\begin{array}{c}\text { Eta cuadrado } \\
\text { parcial }\end{array}$ \\
\hline Modelo corregido & 34895665.819 & 18 & 1938648.101 & 1378854.420 & 0.000 & 0.575 \\
\hline Intersección & 494116.453 & 1 & 494116.453 & 351438.023 & 0.000 & 0.019 \\
\hline Sexo & 51510.896 & 1 & 51510.896 & 36636.884 & 0.000 & 0.002 \\
\hline Edad (categórica) & 69549.658 & 5 & 13909.932 & 9893.374 & 0.000 & 0.003 \\
\hline Nivel de estudios & 47954.862 & 8 & 5994.358 & 4263.459 & 0.000 & 0.002 \\
\hline GSmotivación & 2487360.965 & 1 & 2487360.965 & 1769123.885 & 0.000 & 0.088 \\
\hline GSactividad & 3907907.037 & 1 & 3907907.037 & 2779480.653 & 0.000 & 0.132 \\
\hline GSorg.trabajo & 1252126.761 & 1 & 1252126.761 & 890569.319 & 0.000 & 0.046 \\
\hline GSsalario & 2378246.225 & 1 & 2378246.225 & 1691516.535 & 0.000 & 0.084 \\
\hline Error & 25802458.132 & 18351878 & & & & \\
\hline Total & 1054257987.000 & 18351897 & & & & \\
\hline Total corregida & 60698123.952 & 18351896 & & & & \\
\hline Nota: c. & & & & & \\
\hline
\end{tabular}

Nota: c. modelo con efectos principales.

Variable dependiente: GStrabajoactual. a. $R^{2}\left(\right.$ año 2007) $=0.635, R^{2}\left(\right.$ año 2009) $=0.481, R^{2}($ año 2010) $=0.575$

Tabla 3. Resumen de los modelos lineales, estimaciones del tamaño de los efectos, por años (Modelos grupo 3c). Fuente: elaboración propia a partir de ECVT (Ministerio de Empleo y Seguridad Social: 2007, 2009 y 2010)

Ya que afirmamos que las variables propias del trabajo u organizacionales explican mejor la satisfacción laboral (esta misma idea puede verse en el trabajo de Sánchez-Sellero, Sánchez-Sellero, Cruz-González y Sánchez-Sellero (2014)), en el modelo siguiente incluimos únicamente este tipo de variables pero en mayor número; para establecer una jerarquía entre ellas utilizamos el método de regresión lineal múltiple stepwise, porque cuando existe un elevado número de posibles variables independientes conviene saber cuáles son las variables relevantes. Este método fue estudiado, entre otros, por Derksen y Keselman (1992), Leigh (1988), Thompson (1995) y Thompson (2001).

Partimos, por tanto, de una variable dependiente (GStrabajoactual) y una serie de variables independientes, todas ellas variables que miden el grado de satisfacción subjetivo de cada persona con una serie de aspectos laborales (motivación, actividad, salario, etc.). Pero si la satisfacción laboral depende en gran medida del grado de satisfacción sobre una serie de cuestiones laborales, ¿de qué variables depende más?, ¿es más importante para estar satisfecho laboralmente el grado de satisfacción con el salario o el grado de satisfacción con la actividad? ¿o con la valoración de superiores? En un paso previo a la regresión stepwise aplicamos el análisis de componentes principales (ACP) buscando dos objetivos:

- eliminar la correlación entre las variables independientes,

- reducir el número de variables independientes y convertirlas en factores, que son agrupaciones de las primeras, y así averiguar a continuación cuáles son las variables (o el conjunto de ellas) que repercuten en mayor medida en el grado de satisfacción laboral. 
En la Tabla 4 aparecen las medias y sus tasas de variación tanto para la variable dependiente como para las 14 variables independientes elegidas, todas ellas cuantitativas (toman valores de 0 a 10). Con este análisis damos cumplimiento al objetivo tres de este trabajo, que es la influencia del ciclo económico en las variables de satisfacción. La variable grado satisfacción con el salario -GSsalario- es la segunda con menor puntuación media, indicativo del efecto de la situación de crisis económica. En términos generales, la satisfacción se incrementa con la crisis salvo la satisfacción con horarios, estabilidad y salario; se debe a que esta crisis ha traído aumento de la precariedad laboral y bajada de salarios. En el estudio de Bell y Blanchflower (2011) se señala que los niveles de satisfacción han bajado entre 20072010 debido a la crisis económica en el sur de Europa que trajo consigo altas tasas de desempleo sobre todo entre los grupos más jóvenes de la población activa. Igualmente, Markowits, Boer y Van Dick (2014) demostraron que la crisis tiene efectos negativos sobre los resultados relacionados con el trabajo. Fuera de Europa, Mehri, Iqbal, Hekmat y Ishaq (2011) llegaron a la conclusión de que los trabajadores estudiados redujeron drásticamente la motivación en el trabajo. En nuestro análisis no podemos hablar de lo que sucede con la motivación antes y durante la crisis porque no tenemos el dato de 2007 (no existía esta variable en ese año), y en 2009 y 2010 la cifra se mantuvo.

\begin{tabular}{|c|c|c|c|c|}
\hline Variables & $\begin{array}{c}\text { Año } 2007 \\
\text { Media }\end{array}$ & $\begin{array}{c}\text { Año } 2009 \\
\text { Media }\end{array}$ & $\begin{array}{c}\text { Año } 2010 \\
\text { Media }\end{array}$ & $\begin{array}{c}\text { Tasas Variación } \\
2007-2010(\%)\end{array}$ \\
\hline GStrabajoactual & 7.2 & 7.3 & 7.4 & 2.8 \\
\hline GSposibilidadpromociones & 5.1 & 5.0 & 5.2 & 2.0 \\
\hline GSvaloraciónsuperiores & 6.9 & 7.0 & 7.2 & 4.3 \\
\hline GSactividad & 7.7 & 7.6 & 7.8 & 1.3 \\
\hline GSdesarrollopersonal & 7.3 & 7.5 & 7.6 & 4.1 \\
\hline GSautonomia & 7.3 & 7.5 & 7.5 & 2.7 \\
\hline GSparticipacióndecisiones & 6.6 & 7.0 & 6.9 & 4.5 \\
\hline GSambientetrabajo /GSmotivación* & 7.9 & 7.2 & 7.2 & \\
\hline GSjornada & 6.9 & 7.0 & 7.1 & 2.9 \\
\hline GSflexibilidadhorarios & 6.6 & 6.3 & 6.5 & -1.5 \\
\hline GSvacaciones.permisos & 6.9 & 7.1 & 7.1 & 2.9 \\
\hline GSestabilidad & 7.3 & 7.1 & 7.1 & -2.7 \\
\hline GSsalario & 6.2 & 6.0 & 5.8 & -6.5 \\
\hline GStiempodescanso & 6.4 & 6.5 & 6.7 & 4.7 \\
\hline GSorganizacióntrabajo & 6.7 & 7.1 & 7.1 & 6.0 \\
\hline
\end{tabular}

Nota: variable dependiente: GStrabajoactual

*En 2007 la variable es GSambientetrabajo, y en 2009 y 2010 es GSmotivación

Tabla 4. Estadísticos descriptivos por años y tasas de variación 2007-2010 (variables propias del trabajo). Fuente: elaboración propia a partir de ECVT (Ministerio de Empleo y Seguridad Social: 2007,2009 y 2010). 
Recordemos que el segundo objetivo que nos habíamos marcado era englobar las variables relativas al trabajo en grupos homogéneos. El análisis de componentes principales (Everitt \& Dunn, 2001; Bartholomew, Steele, Moustaki \& Galbraith, 2008) se puede aplicar a un grupo de variables para descubrir qué conjuntos de ellas forman subconjuntos coherentes que son independientes entre sí. Se utiliza a menudo en la reducción de datos para identificar un pequeño número de factores que explica la mayor parte de la varianza observada partiendo de un número mucho mayor de variables iniciales.

Para los 3 años estudiados, el determinante de la matriz de correlaciones de las variables es casi cero (véase Tabla 5). El valor de KMO (medida de Kaiser-Meyer-Olkin) es $>0.500$ (bueno); valores superiores a 0.7 son aceptables (Norman \& Streiner, 2008). Y la prueba de Bartlett tiene un $\mathrm{p}=0.000<0.05$ que permite rechazar la hipótesis de matriz identidad. Un estudio ACP en satisfacción laboral se encuentra en Platis, Reklitis y Zimeras (2015).

\begin{tabular}{|c|l|r|r|r|}
\cline { 2 - 4 } \multicolumn{2}{l|}{} & Año 2007 & Año 2009 & Año 2010 \\
\hline Determinante de la matriz de correlaciones & 0.003 & 0.004 & 0.003 \\
\hline Medida de adecuación muestral de Kaiser-Meyer-Olkin & 0.926 & 0.902 & 0.899 \\
\hline \multirow{3}{*}{\begin{tabular}{c} 
Prueba de esfericidad de Bartlett \\
\cline { 2 - 5 }
\end{tabular}} & Chi-cuadrado aproximado & 33516.311 & 34954.709 & 33684.295 \\
\cline { 2 - 5 } & gl & 91 & 91 & 91 \\
\cline { 2 - 5 } & Sig. & 0.000 & 0.000 & 0.000 \\
\hline
\end{tabular}

Tabla 5. Diagnóstico de las condiciones de los análisis de componentes principales, por años. Fuente: elaboración propia a partir de ECVT (Ministerio de Empleo y Seguridad Social: 2007, 2009 y 2010)

Mediante el sistema de rotación Quartimax y ordenando los coeficientes por tamaño extraemos 5, 7 y 6 factores en los análisis de 2007, 2009 y 2010 respectivamente (véase Tabla 6). De este modo, el porcentaje de variabilidad explicado por los factores extraídos está entre 70-80\%. La matriz de componentes rotados aparece en la tabla 6. Los factores tienen media 0 y desviación típica 1 , y las correlaciones entre las variables explicativas son 0. Vemos que GSsalario es un factor que no se agrupa con ninguna otra variable y que ocupa el último o penúltimo lugar en la explicación de la variable GStrabajoactual. En la diagonal hemos resaltado en negrilla las saturaciones $>0.500$ que indican una asociación elevada. 


\begin{tabular}{|c|c|c|c|c|c|c|c|}
\hline \multicolumn{6}{|c|}{ AÑO 2007} & & \\
\hline \multirow[t]{2}{*}{ Variables } & \multicolumn{7}{|c|}{ Componente } \\
\hline & 1 & 2 & 3 & 4 & 5 & & \\
\hline GSparticipac.decisiones & 0.803 & 0.022 & 0.025 & -0.247 & -0.078 & & \\
\hline GSdesarrollopersonal & 0.787 & 0.051 & 0.049 & 0.178 & 0.106 & & \\
\hline GSvaloraciónsuperiores & 0.783 & 0.119 & -0.079 & 0.102 & 0.093 & & \\
\hline GSautonomia & 0.780 & 0.105 & 0.110 & -0.094 & -0.094 & & \\
\hline GSorganizacióntrabajo & 0.756 & 0.149 & 0.012 & 0.055 & 0.000 & & \\
\hline GSposibilidadpromociones & 0.663 & 0.057 & 0.089 & -0.433 & 0.116 & & \\
\hline GSactividad & 0.651 & 0.035 & 0.188 & 0.443 & 0.159 & & \\
\hline GSflexibilidadhorarios & 0.395 & 0.738 & 0.063 & -0.059 & -0.018 & & \\
\hline GStiempodescanso & 0.383 & 0.730 & 0.082 & -0.011 & 0.003 & & \\
\hline GSjornada & 0.356 & 0.684 & 0.114 & 0.153 & 0.254 & & \\
\hline GSestabilidad & 0.438 & 0.074 & 0.786 & 0.030 & 0.081 & & \\
\hline GSvacaciones.permisos & 0.377 & 0.388 & 0.665 & 0.045 & 0.014 & & \\
\hline GSambientetrabajo & 0.575 & 0.125 & 0.080 & 0.589 & -0.051 & & \\
\hline GSsalario & 0.437 & 0.224 & 0.098 & -0.025 & 0.818 & & \\
\hline \multicolumn{8}{|c|}{ AÑO 2009} \\
\hline \multirow[t]{2}{*}{ Variables } & \multicolumn{7}{|c|}{ Componente } \\
\hline & 1 & 2 & 3 & 4 & 5 & 6 & 7 \\
\hline GSactividad & 0.827 & 0.111 & 0.205 & 0.218 & 0.155 & 0.155 & 0.120 \\
\hline GSdesarrollopersonal & 0.814 & 0.097 & 0.273 & 0.194 & 0.134 & 0.166 & 0.164 \\
\hline GSmotivación & 0.520 & 0.148 & 0.427 & 0.374 & 0.098 & 0.249 & 0.209 \\
\hline GSflexibilidadhorarios & 0.055 & 0.772 & 0.259 & 0.047 & 0.097 & 0.059 & 0.217 \\
\hline GStiempodescanso & 0.035 & 0.740 & 0.080 & 0.193 & 0.182 & 0.182 & 0.071 \\
\hline GSjornada & 0.313 & 0.667 & -0.025 & 0.108 & 0.297 & 0.184 & 0.009 \\
\hline GSparticipac.decisiones & 0.173 & 0.071 & 0.809 & 0.213 & 0.093 & 0.152 & 0.199 \\
\hline GSautonomia & 0.256 & 0.143 & 0.793 & 0.130 & 0.180 & 0.097 & 0.097 \\
\hline GSorganizacióntrabajo & 0.185 & 0.093 & 0.149 & 0.822 & 0.111 & 0.151 & 0.134 \\
\hline GSvaloraciónsuperiores & 0.172 & 0.124 & 0.174 & 0.769 & 0.125 & 0.127 & 0.245 \\
\hline GSestabilidad & 0.083 & 0.050 & 0.147 & 0.094 & 0.857 & 0.138 & 0.169 \\
\hline GSvacaciones.permisos & 0.129 & 0.300 & 0.086 & 0.120 & 0.769 & 0.146 & 0.047 \\
\hline GSsalario & 0.091 & 0.094 & 0.078 & 0.095 & 0.127 & 0.960 & 0.139 \\
\hline GSposibilidadpromociones & 0.078 & 0.066 & 0.087 & 0.150 & 0.092 & 0.135 & 0.953 \\
\hline \multicolumn{8}{|c|}{ AÑO 2010} \\
\hline \multirow[t]{2}{*}{ Variables } & \multicolumn{7}{|c|}{ Componente } \\
\hline & 1 & 2 & 3 & 4 & 5 & 6 & \\
\hline GSactividad & 0.890 & 0.140 & 0.038 & 0.070 & 0.003 & 0.042 & \\
\hline GSdesarrollopersonal & 0.883 & 0.117 & 0.058 & 0.065 & 0.085 & 0.060 & \\
\hline GSmotivación & 0.741 & 0.168 & 0.287 & 0.084 & 0.198 & 0.088 & \\
\hline GSflexibilidadhorarios & 0.119 & 0.810 & 0.148 & 0.019 & 0.249 & -0.013 & \\
\hline GStiempodescanso & 0.208 & 0.754 & 0.090 & 0.178 & 0.029 & 0.032 & \\
\hline GSjornada & 0.301 & 0.714 & 0.024 & 0.179 & -0.131 & 0.139 & \\
\hline GSposibilidadpromociones & 0.126 & 0.094 & 0.738 & 0.026 & 0.277 & 0.185 & \\
\hline GSvaloraciónsuperiores & 0.479 & 0.147 & 0.658 & 0.116 & -0.013 & -0.020 & \\
\hline GSorganizacióntrabajo & 0.501 & 0.160 & 0.656 & 0.095 & -0.106 & -0.014 & \\
\hline GSestabilidad & 0.215 & 0.171 & 0.129 & 0.843 & 0.120 & 0.089 & \\
\hline GSvacaciones.permisos & 0.241 & 0.413 & 0.046 & 0.714 & 0.018 & 0.044 & \\
\hline GSparticipacióndecisiones & 0.447 & 0.124 & 0.213 & 0.104 & 0.717 & 0.042 & \\
\hline GSautonomia & 0.568 & 0.163 & 0.074 & 0.130 & 0.619 & 0.018 & \\
\hline GSsalario & 0.267 & 0.181 & 0.182 & 0.142 & 0.049 & 0.900 & \\
\hline
\end{tabular}

Nota: Método de extracción: Análisis de componentes principales.

a Método de rotación: Normalización Quartimax con Kaiser

Tabla 6. Matriz de componentes rotados (Análisis ACP) ${ }^{\mathrm{a}}$, por años. Fuente: elaboración propia a partir de ECVT (Ministerio de Empleo y Seguridad Social: 2007, 2009 y 2010) 
Por último, y como cuarto objetivo, estableceremos una jerarquía para saber cuáles son las variables independientes y/o factores obtenidos en el análisis ACP que ejercen mayor influencia en la satisfacción laboral. Haremos la regresión stepwise de dos formas: la primera con las variables originales referidas al trabajo, y la segunda con los factores extraídos del análisis de componentes principales, ACP. Un análisis de regresión realizado a partir de los factores extraídos de un ACP puede verse también en Aizawa, Kishi, Aizawa, Hirata, Ishii y Yonemitsu (2015), y un análisis de ACP en un estudio de satisfacción laboral se encuentra en Leung, Rioseco y Munro (2015). El método de regresión stepwise resulta útil para explicar una variable dependiente (en este caso, GStrabajoactual) a partir de varias variables independientes potenciales (predictores) en el caso de que no exista una teoría que permita seleccionar de antemano un subconjunto de predictores para evaluar el modelo. Básicamente, se trata de que el programa informático con el cual se realiza el análisis estadístico, seleccione aquel conjunto de variables que contribuyan de forma significativa al ajuste del modelo. Sólo se incorporan las variables que son predictores significativos de la satisfacción laboral. La contribución de cada variable se establece contrastando, a partir del coeficiente de correlación parcial, la hipótesis de independencia entre esa variable y la variable dependiente. Encontramos aplicaciones del modelo de regresión stepwise en el ámbito de la satisfacción laboral en los trabajos de Lloréns y Senise (1996), García, Barbero, Ávila y García (2003) y Goetz et al. (2015), aunque con distintas variables en el modelo.

En este método stepwise se adopta el criterio de que una variable es incorporada al modelo si el nivel crítico asociado a su coeficiente de correlación parcial al contrastar la hipótesis de independencia es menor que 0.05 (probabilidad de entrada). Y queda fuera del modelo si ese nivel crítico es mayor que 0.10 (probabilidad de salida). Por razones de espacio, en la Tabla 7 aparece solo el resumen de los modelos de regresión stepwise para distintos años a partir de los factores de ACP. La Tabla 7 recoge el valor de $\mathrm{R}, \mathrm{R}^{2}$ en cada paso. El error típico de la estimación va disminuyendo (pasa de 1.35612 a 1.06569 en 2010). Una forma de valorar el efecto resultante de aplicar estos criterios de selección consiste en observar el cambio que se va produciendo en $\mathrm{R}^{2}$ a medida que se van incorporando variables al modelo. Un cambio grande en $\mathrm{R}^{2}$ indica que la variable en cuestión contribuye de forma importante a explicar lo que ocurre con la variable dependiente. En la Tabla 7 también aparece el estadístico $\mathrm{F}$, que contrasta la hipótesis de que el cambio en $\mathrm{R}^{2}$ es cero en la población, y el nivel crítico asociado al estadístico F. Las 6 variables independientes (son los factores de ACP, año 2010) explican un 63.0\% de la varianza de la variable GStrabajoactual. El estadístico de Durbin-Watson vale 1.973 con lo que se asume independencia entre los residuos; éste es uno de los supuestos en que se basa el modelo de regresión lineal. 


\begin{tabular}{|c|c|c|c|c|c|c|c|c|c|c|}
\hline \multicolumn{11}{|c|}{ Año 2007} \\
\hline \multirow[b]{2}{*}{ Modelo } & \multirow[b]{2}{*}{$\mathbf{R}$} & \multirow[b]{2}{*}{$\mathbf{R}^{2}$} & \multirow[b]{2}{*}{$\begin{array}{c}\mathbf{R}^{2} \\
\text { corregida }\end{array}$} & \multirow[b]{2}{*}{$\begin{array}{l}\text { Error típ. de } \\
\text { la estimación }\end{array}$} & \multicolumn{5}{|c|}{ Estadísticos de cambio } & \multirow[b]{2}{*}{$\begin{array}{l}\text { Durbin- } \\
\text { Watson }\end{array}$} \\
\hline & & & & & $\begin{array}{c}\text { Cambio en } \\
\mathbf{R}^{2}\end{array}$ & $\begin{array}{c}\text { Cambio en } \\
F\end{array}$ & gl1 & g12 & $\begin{array}{l}\text { Sig. del } \\
\text { cambio } \\
\text { en } F\end{array}$ & \\
\hline 1 & $0.751^{\mathrm{a}}$ & 0.563 & 0.563 & 1.15457 & 0.563 & 7295.681 & 1 & 5654 & 0.000 & \\
\hline 2 & $0.804^{\mathrm{b}}$ & 0.646 & 0.646 & 1.04008 & 0.082 & 1314.313 & 1 & 5653 & 0.000 & \\
\hline 3 & $0.833^{\mathrm{c}}$ & 0.694 & 0.694 & 0.96646 & 0.048 & 895.057 & 1 & 5652 & 0.000 & \\
\hline 4 & $0.853^{\mathrm{d}}$ & 0.727 & 0.727 & 0.91284 & 0.033 & 684.388 & 1 & 5651 & 0.000 & \\
\hline 5 & $0.862^{\mathrm{e}}$ & 0.743 & 0.742 & 0.88670 & 0.015 & 339.182 & 1 & 5650 & 0.000 & 2.034 \\
\hline \multicolumn{11}{|c|}{ Año 2009} \\
\hline \multirow[b]{2}{*}{ Modelo } & \multirow[b]{2}{*}{$\mathbf{R}$} & \multirow[b]{2}{*}{$\mathbf{R}^{2}$} & \multirow[b]{2}{*}{$\begin{array}{c}\mathbf{R}^{2} \\
\text { corregida }\end{array}$} & \multirow[b]{2}{*}{$\begin{array}{l}\text { Error típ. de } \\
\text { la estimación }\end{array}$} & \multicolumn{5}{|c|}{ Estadísticos de cambio } & \multirow[b]{2}{*}{$\begin{array}{l}\text { Durbin- } \\
\text { Watson }\end{array}$} \\
\hline & & & & & $\begin{array}{c}\text { Cambio en } \\
\mathbf{R}^{2}\end{array}$ & $\begin{array}{c}\text { Cambio en } \\
\text { F }\end{array}$ & gl1 & g12 & $\begin{array}{l}\text { Sig. del } \\
\text { cambio } \\
\text { en F }\end{array}$ & \\
\hline 1 & $0.433^{\mathrm{a}}$ & 0.188 & 0.188 & 1.63824 & 0.188 & 1449.092 & 1 & 6272 & 0.000 & \\
\hline 2 & $0.559^{\mathrm{b}}$ & 0.313 & 0.313 & 1.50697 & 0.125 & 1141.317 & 1 & 6271 & 0.000 & \\
\hline 3 & $0.627^{\mathrm{c}}$ & 0.393 & 0.393 & 1.41621 & 0.080 & 830.523 & 1 & 6270 & 0.000 & \\
\hline 4 & $0.655^{\mathrm{d}}$ & 0.429 & 0.429 & 1.37368 & 0.036 & 395.189 & 1 & 6269 & 0.000 & \\
\hline 5 & $0.678^{\mathrm{e}}$ & 0.460 & 0.459 & 1.33638 & 0.031 & 355.846 & 1 & 6268 & 0.000 & \\
\hline 6 & $0.700^{\mathrm{f}}$ & 0.490 & 0.489 & 1.29873 & 0.030 & 369.689 & 1 & 6267 & 0.000 & \\
\hline 7 & $0.716^{\mathrm{g}}$ & 0.513 & 0.512 & 1.26930 & 0.023 & 295.009 & 1 & 6266 & 0.000 & 1.978 \\
\hline \multicolumn{11}{|c|}{ Año 2010} \\
\hline \multirow[b]{2}{*}{ Modelo } & \multirow[b]{2}{*}{$\mathbf{R}$} & \multirow[b]{2}{*}{$\mathbf{R}^{2}$} & \multirow[b]{2}{*}{$\begin{array}{c}\mathbf{R}^{2} \\
\text { corregida }\end{array}$} & \multirow[b]{2}{*}{$\begin{array}{l}\text { Error típ. de } \\
\text { la estimación }\end{array}$} & \multicolumn{5}{|c|}{ Estadísticos de cambio } & \multirow[b]{2}{*}{$\begin{array}{l}\text { Durbin- } \\
\text { Watson }\end{array}$} \\
\hline & & & & & $\begin{array}{c}\text { Cambio en } \\
\mathbf{R}^{2}\end{array}$ & $\begin{array}{l}\text { Cambio en } \\
F\end{array}$ & g11 & g12 & $\begin{array}{l}\text { Sig. del } \\
\text { cambio } \\
\text { en F }\end{array}$ & \\
\hline 1 & $0.633^{\mathrm{a}}$ & 0.400 & 0.400 & 1.35612 & 0.400 & 3897.721 & 1 & 5839 & 0.000 & \\
\hline 2 & $0.699^{\mathrm{b}}$ & 0.489 & 0.488 & 1.25248 & 0.088 & 1007.323 & 1 & 5838 & 0.000 & \\
\hline 3 & $0.749^{\mathrm{c}}$ & 0.561 & 0.561 & 1.15984 & 0.073 & 970.791 & 1 & 5837 & 0.000 & \\
\hline 4 & $0.773^{\mathrm{d}}$ & 0.598 & 0.598 & 1.11022 & 0.037 & 534.471 & 1 & 5836 & 0.000 & \\
\hline 5 & $0.791^{\mathrm{e}}$ & 0.626 & 0.625 & 1.07198 & 0.027 & 424.784 & 1 & 5835 & 0.000 & \\
\hline 6 & $0.794^{\mathrm{f}}$ & 0.630 & 0.630 & 1.06569 & 0.004 & 70.030 & 1 & 5834 & 0.000 & 1.973 \\
\hline
\end{tabular}

Año 2007

a. Variables predictoras: (Constante), factor 1

b. Variables predictoras: (Constante), a la anterior se añade el factor 2

c. Variables predictoras: (Constante), a las anteriores se añade el factor 5

d. Variables predictoras: (Constante), a las anteriores se añade el factor 3

e. Variables predictoras: (Constante), a las anteriores se añade el factor 4

Año 2009

a. Variables predictoras: (Constante), factor 1

b. Variables predictoras: (Constante), a la anterior se añade el factor 4

c. Variables predictoras: (Constante), a las anteriores se añade el factor 6

d. Variables predictoras: (Constante), a las anteriores se añade el factor 3

e. Variables predictoras: (Constante), a las anteriores se añade el factor 5

f. Variables predictoras: (Constante), a las anteriores se añade el factor 2

g. Variables predictoras: (Constante), a las anteriores se añade el factor 7

Año 2010

a. Variables predictoras: (Constante), factor 1

b. Variables predictoras: (Constante), a la anterior se añade el factor 3

c. Variables predictoras: (Constante), a las anteriores se añade el factor 2

d. Variables predictoras: (Constante), a las anteriores se añade el factor 4

e. Variables predictoras: (Constante), a las anteriores se añade el factor 6

f. Variables predictoras: (Constante), a las anteriores se añade el factor 5

h. Variable dependiente: GStrabajoactual

Tabla 7. Resumen de los modelos de regresión stepwise obtenidos a partir de los factores de los análisis de componentes principales $(\mathrm{ACP})^{\mathrm{h}}$, por años. Fuente: elaboración propia a partir de ECVT (Ministerio de

Empleo y Seguridad Social: 2007, 2009 y 2010). 
$\mathrm{Al}$ ir incorporando los factores, se va produciendo una mejora en el modelo, ya que se va incrementando la suma de cuadrados de la regresión en los distintos pasos, mientras que la suma de cuadrados residual va disminuyendo.

Nuestro siguiente paso consiste en volver a hacer la regresión stepwise pero ahora con las variables originales, es decir, con las 14 variables de satisfacción relativas al trabajo. Por último, compararemos estos resultados con los obtenidos en la regresión stepwise realizada a partir de los factores extraídos del análisis ACP; en la Tabla 8 aparece una comparativa de ambos procesos (con ACP y sin ACP). La jerarquía de las variables así como los $\mathrm{R}^{2}$ en ambos procesos de regresión para los 3 años estudiados aparecen también en la Tabla 8.

\begin{tabular}{|c|c|c|c|c|c|}
\hline \multicolumn{2}{|l|}{ Año 2007} & \multicolumn{2}{|l|}{ Año 2009} & \multicolumn{2}{|l|}{ Año 2010} \\
\hline Sin ACP & Con ACP & Sin ACP & Con ACP & Sin ACP & Con ACP \\
\hline $\begin{array}{l}\text { Jerarquía } \\
\text { variables }\end{array}$ & $\begin{array}{l}\text { Jerarquía } \\
\text { factores }\end{array}$ & $\begin{array}{l}\text { Jerarquía } \\
\text { variables }\end{array}$ & $\begin{array}{l}\text { Jerarquía } \\
\text { factores }\end{array}$ & $\begin{array}{l}\text { Jerarquía } \\
\text { variables }\end{array}$ & $\begin{array}{l}\text { Jerarquía } \\
\text { factores }\end{array}$ \\
\hline GSdesarrollopersonal & Factor 1 & GSmotivación & Factor 1 & GSmotivacion & Factor 1 \\
\hline GSsalario & Factor 2 & GSactividad & Factor 4 & GSactividad & Factor 3 \\
\hline GSorganizaciontrabajo & Factor 5 & GSorganizaciontrabajo & Factor 6 & GSorganizaciontrabajo & Factor 2 \\
\hline GSjornada & Factor 3 & GSsalario & Factor 3 & GSsalario & Factor 4 \\
\hline GSactividad & Factor 4 & GSjornada & Factor 5 & GSjornada & Factor 6 \\
\hline GSautonomia & & GSvaloracionsuperiores & Factor 2 & GSvaloracionsuperiores & Factor 5 \\
\hline GSambientetrabajo & & GSvacacionespermisos & Factor 7 & GSestabilidad & \\
\hline GSposibilidadpromoc. & & GSdesarrollopersonal & & GSdesarrollopersonal & \\
\hline GSvacacionespermisos & & GSparticipacdecisiones & & GSvacacionespermisos & \\
\hline GSflexibilidadhorarios & & GSestabilidad & & GSflexibilidadhorarios & \\
\hline GSestabilidad & & & & GSautonomia & \\
\hline GSvaloracionsuperiores & & & & GStiempodescanso & \\
\hline GSparticipac.decisiones & & & & GSposibilidadpromoc. & \\
\hline GStiempodescanso & & & & & \\
\hline $\mathbf{R}^{2}$ & $\mathbf{R}^{2}$ & $\mathbf{R}^{2}$ & $\mathbf{R}^{2}$ & $\mathbf{R}^{2}$ & $\mathbf{R}^{2}$ \\
\hline 0.744 & 0.743 & 0.521 & 0.513 & 0.634 & 0.630 \\
\hline
\end{tabular}

Variable dependiente: GStrabajoactual

Tabla 8. Jerarquía de las variables y bondad de los modelos de regresión stepwise obtenidos sin componentes principales y con componentes principales, por años. Fuente: elaboración propia a partir de ECVT

(Ministerio de Empleo y Seguridad Social: 2007, 2009 y 2010) 


\section{Discusión}

En la Tabla 1 podemos ver que los modelos mejoran sensiblemente si las variables independientes son las relativas al trabajo -misma idea que en Sánchez-Sellero et al. (2014). En este sentido (Tabla 3), la estimación de la proporción de la variable dependiente que está explicada por cada efecto (estadístico eta cuadrado parcial) es mucho mayor en estas variables que en las variables personales, tanto en el año de crecimiento (2007) como en años de crisis (2009 y 2010).

Un hecho que nos parece muy significativo es que repasadas las tasas de variación de los grados de satisfacción medios en el período 2007-2010 (Tabla 4), aumentan todas excepto GSflexibilidadhorarios, GSestabilidad y GSsalario. La primera, al ser de menor cuantía (-1.5\%), le restamos importancia, pero estabilidad $(-2.7 \%)$ y sobre todo salario $(-6.5 \%)$ tienen explicación ya que en esta crisis la estabilidad laboral se ha visto mermada y las medidas económicas han traído rebajas salariales.

En el análisis de componentes principales (ACP), las matrices de componentes rotados (Tabla 6) para los años de crisis -2009 y 2010- tienen muchas similitudes. El factor 1 engloba los grados de satisfacción con la actividad, desarrollo personal y motivación. El factor 2 engloba flexibilidad de horarios, tiempos de descanso y jornada (variables que tienen relación entre sî). Otro factor agrupa estabilidad, y vacaciones y permisos (en 2007, 2009 y 2010); se debe a que los trabajos de corta duración no dan derecho a vacaciones. Otro factor está formado únicamente por la variable GSsalario.

En la regresión stepwise hecha con los factores (Tabla 7), el factor 1 -que corresponde a actividad, desarrollo personal y motivación- es el que tiene mayor influencia en la satisfacción laboral. El factor del salario (factor 5, factor 6 y factor 6 en 2007, 2009 y 2010 respectivamente) ocupa un lugar intermedio en los modelos por grado de explicación. Este resultado es compatible con las investigaciones ya citadas, de autores como Judge et al. (2010), Böckerman et al. (2011) y Tremblay et al. (2012), en las que la remuneración no juega un papel decisivo en la satisfacción.

Al comparar con las regresiones stepwise hechas con las variables originales (Tabla 8), de las 14 variables independientes, el salario ocupa el $2^{\circ}$ o $4^{\circ}$ puesto. Obtenemos todos los coeficientes positivos y significativos. En el año 2009 hay 4 variables que salen del modelo, con lo que quedan 10 variables independientes. Destacamos dos ideas importantes: primero, la ordenación o jerarquía de las variables en la regresión no es la misma si se aplica el análisis ACP o si no se aplica, lógicamente porque con el ACP se elimina la interacción de las variables implicadas; segundo, la bondad de los modelos de regresión stepwise hechos con las variables originales y hechos con los factores de ACP nos arroja resultados muy parecidos $\left(\mathrm{R}^{2}=0.634\right.$ y $\mathrm{R}^{2}=0.630$ en 2010). Los porcentajes de explicación en los 
modelos con ACP, 74\%, 51\% y 63\% en cada uno de los años, los consideramos ampliamente satisfactorios.

\section{Conclusiones}

A la vista del marco teórico sobre el tema, podemos afirmar que los factores que pueden influir en la satisfacción de los trabajadores son muchos: características personales de cada trabajador, características inherentes al trabajo u organizacionales, factores del entorno, el contexto macroeconómico e incluso el marco político general.

Los datos utilizados en este trabajo son los microdatos de la ECVT de los años 2007, 2009 y 2010; en 2016 la última ECVT es de 2010. Aplicamos distintas metodologías: modelos lineales generales (ANOVA), análisis de componentes principales (ACP) y modelo de regresión lineal múltiple stepwise, con la finalidad de explicar la variable GStrabajoactual. Esta combinación de técnicas estadísticas en el estudio de la satisfacción laboral es totalmente novedosa; además, se aplican en años correspondientes a distinto ciclo económico.

Aunque de los factores que inciden en la satisfacción laboral se puede hacer un estudio mucho más pormenorizado, no es ese nuestro propósito en este trabajo. De las características personales hemos incluido las 3 que consideramos más destacadas y de las características organizacionales incorporamos 14 que consideramos relevantes. Nuestro objetivo era múltiple: Primero, averiguar qué tipo de características influyen en mayor grado en la satisfacción laboral; Con la aplicación de modelos lineales (ANOVA) llegamos a la conclusión de que aunque las variables son significativas igualmente, las variables referidas al trabajo proporcionan mejores resultados en la explicación de la satisfacción laboral. Hay estudios empíricos que llegan a esta misma conclusión, aunque las variables del modelo no sean exactamente las mismas.

Segundo. Una vez que comprobamos que ese grupo corresponde a las características inherentes al trabajo, agrupamos esas variables en grupos homogéneos llamados factores mediante el análisis de componentes principales (ACP). Durante los años de crisis hay 4 factores muy claros y coherentes: el factor 1 agrupa actividad, desarrollo personal y motivación; otro factor aglutina flexibilidad de horarios, tiempo de descanso y jornada; un tercer factor está formado por estabilidad y vacaciones-permisos; y un cuarto factor son los salarios. 
Tercero. Un hecho destacado es que los grados de satisfacción medios vinculados con el trabajo aumentan de manera generalizada en periodo de crisis, con 3 excepciones (satisfacción con la estabilidad, salarios y flexibilidad de horarios). La disminución de GSestabilidad y GSsalario se debe a la situación económica vigente marcada por un aumento de la precariedad laboral y la reducción salarial. En términos generales, los contratos laborales en este período se caracterizan porque son menos estables y los salarios son más bajos.

Cuarto. Tratamos de averiguar cuáles son las variables y/o factores que ejercen mayor contribución en nuestra variable dependiente, GStrabajoactual. Comprobamos que las variables más relevantes son las mismas en período de crisis que en crecimiento, es decir, no dependen del ciclo económico, aunque las jerarquías de las variables tienen más similitudes en los años de crisis $(2009,2010)$ que en el año de crecimiento de nuestro análisis (2007). En la regresión lineal múltiple stepwise hecha con los factores del análisis ACP, el factor 1 (actividad, desarrollo personal y motivación) es el que aporta mayor explicación a la variable dependiente.

De los resultados de distintos estudios se deduce que a mayor satisfacción mayor rendimiento laboral, y por tanto, tendencia a mejorar la productividad en el trabajo y el grado de competitividad de la empresa. Todo ello debería servir de reflexión a empresas y a instituciones públicas y privadas para contemplar distintas medidas encaminadas a fomentar el avance en este tipo de satisfacción; estas actuaciones podrían concretarse en cursos de formación dirigidos a empresarios y empleadores con la finalidad de mejorar la organización en el trabajo, en los turnos, permisos, etc. También serían de provecho cursos para empresarios y directivos, en aras a mejorar la motivación y el desarrollo personal de los trabajadores, en definitiva, para que el clima laboral mejore. Mandar y motivar a la vez no es tarea fácil, y, si se consigue, además de reflejar una buena calidad profesional y humana, reportará beneficios al trabajador ya que estará más satisfecho, a la empresa por su mayor rendimiento, e incluso al directivo por la satisfacción de un trabajo bien hecho.

En nuestro ánimo está continuar este estudio con datos más recientes, sin embargo, en la actualidad no es posible debido a que en 2016 la última encuesta ECVT (2010) es la que hemos utilizado para elaborar este trabajo. Una hipotética línea de investigación sería analizar la satisfacción laboral no solo antes y durante la crisis de 2008, sino también después de ella, es decir, cogiendo el ciclo económico completo.

Aunque el concepto de satisfacción en el trabajo es universal, definido desde distintas vertientes por diferentes autores, el estudio en un país y en un momento económico concreto tiene connotaciones 
propias. En España, en 2016 y aunque los indicadores económicos están mejorando, las cifras de desempleo siguen siendo altas; ante esta situación, las exigencias de los trabajadores son menores debido a que es preferible tener un empleo en inferiores condiciones y por el que se cobre menos, que por el contrario, no tenerlo.

\section{Agradecimientos}

Los autores agradecen los fondos recibidos de la Universidad de Zaragoza (proyecto UZ2016-SOC-01) y del Gobierno de Aragón y FEDER (proyecto S125: Grupo de Investigación Compete).

\section{Referencias}

Aizawa, F., Kishi, M., Aizawa, Y., Hirata, S., Ishii, T., \& Yonemitsu, M. (2015). Targeting individual empowerment to raise leaders for local oral health promotion in Japan. Asia-Pacific Journal of Public Health, 27(2), 2305-2315. http://dx.doi.org/10.1177/1010539511428669

Allen, J., \& Van der Velden, R. (2001). Educational mismatches versus skill mismatches: Effects on wages, job satisfaction, and on-the-job search. Oxford Economic Papers, 53(3), 434-452. http://dx.doi.org/10.1093/oep/53.3.434

Alnıaçı, Ü., Alnıaçı, E., Akçin, K., \& Erat, S. (2012). Relationships between career motivation, affective commitment and job satisfaction. Procedia-Social and Behavioral Sciences, 58, 355-362. http://dx.doi.org/10.1016/j.sbspro.2012.09.1011

Aziri, B. (2011). Job satisfaction: A literature review. Management research and practice, 3(4), 77-86.

Bartholomew, D.J., Steele, F., Moustaki, I., \& Galbraith, J.I. (2008). Analysis of Multivariate Social Science Data (2nd edition). Chapman and Hall: CRC.

Bell, D.N.F., \& Blanchflower, D.G. (2011). The crisis, policy reactions and attitudes to globalization and jobs. In M. Bacchetta \& M. Jansen (Eds.), Making globalization socially sustainable (pp. 85-118). Geneva: ILO \& WTO.

Bellou, V. (2010). Organizational culture as a predictor of job satisfaction: The role of gender and age. Career Development International, 15(1), 4-19. http://dx.doi.org/10.1108/13620431011020862 
Böckerman, P., Ilmakunnas, P. \& Johansson, E. (2011). Job Security and Employee Well-being: Evidence from Matched Survey and Register Data. Labour Economics, 18(4), 547-554. http://dx.doi.org/10.1016/j.labeco.2010.12.011

Bòria-Reverter, S., Crespi-Vallbona, M., \& Mascarilla-Miró, O. (2012). Variables determinantes de la satisfacción laboral en España. Cuadernos de Economía,35(97), 9-16. http://dx.doi.org/10.1016/S02100266(12)70018-3

Boumans, N.P., De Jong, A.H., \& Janssen, S.M. (2011). Age-differences in work motivation and job satisfaction. The influence of age on the relationships between work characteristics and workers' outcomes. The International Journal of Aging and Human Development, 73(4), 331-350. http://dx.doi.org/10.2190/AG.73.4.d

Bowling, N.A. (2010). Effects of job satisfaction and conscientiousness on extra-role behaviors. Journal of Business and Psychology, 25(1), 119-130. http://dx.doi.org/10.1007/s10869-009-9134-0

Card, D., Mas, A., Moretti, E., \& Saez, E. (2012). Inequality at Work: The Effect of Peer Salaries on Job Satisfaction. American Economic Review, American Economic Association, 102(6), 2981-3003. http://dx.doi.org/10.1257/aer.102.6.2981

Carrillo-García, C., Solano-Ruíz, M.D.C., Martínez-Roche, M.E., \& Gómez-García, C.I. (2013). Job satisfaction among health care workers: The role of gender and age. Revista latino-americana de enfermagem, 21(6), 1314-1320. http://dx.doi.org/10.1590/0104-1169.3224.2369

Chiang, M., Martín, M.J., \& Núñez, A. (2010). Relaciones entre el clima organizacional y la satisfacción laboral. Madrid: Universidad Pontificia Comillas.

Clark, A., Oswald, A., \& Warr, P. (2011). Is job satisfaction U-shaped in age?. Journal of Occupational and Organizational Psychology, 69(1), 57-81. http://dx.doi.org/10.1111/j.2044-8325.1996.tb00600.x

Cohrs, C., Abele, A.E., \& Dette, D.E. (2006). Integrating situational and dispositional determinants of job satisfaction: Findings from three samples of professionals. The Journal of Psychology, 140(4), 363-995. http://dx.doi.org/10.3200/JRLP.140.4.363-395

Cuadra-Peralta, A.A., \& Veloso-Besio, C.B. (2010). Grado de supervisión como variable moderadora entre liderazgo y satisfacción, motivación y clima organizacional. Ingeniare. Revista chilena de ingeniería,18(1), 15-25. http://dx.doi.org/10.4067/S0718-33052010000100003

Danish, R.Q., \& Usman, A. (2010). Impact of reward and recognition on job satisfaction and motivation: An empirical study from Pakistan. International Journal of Business and Management,5(2), 159-167. http://dx.doi.org/10.5539/ijbm.v5n2p159 
Derksen, S., \& Keselman, H.J. (1992). Backward, forward and stepwise automated subset-selection algorithms: frequency of obtaining authentic and noise variables. British Journal of Mathematical and Statistical Psychology,45, 265-282. http://dx.doi.org/10.1111/j.2044-8317.1992.tb00992.x

Donohue, S.M., \& Heywood, J.S. (2004). Job satisfaction and gender: an expanded specification from the NLSY. International Journal of Manpower,25(2), 211-238. http://dx.doi.org/10.1108/01437720410536007

Dueñas, D., Iglesias, C., \& Llorente, R. (2010). Job quality, job satisfaction and services in Spain. Journal of Innovation Economics,1(5), 145-166.

ECVT, Encuesta de calidad de vida en el trabajo. (2010). Ministerio de Empleo y Seguridad Social, Madrid.

Everitt, B.S., \& Dunn, G. (2001). Applied Multivariate Data Analysis (2nd edition). London: Arnold. http://dx.doi.org/10.1002/9781118887486

Franek, M., \& Vecera, J. (2008). Personal characteristics and job satisfaction. E+M Ekonomie a Management,(4), 63-76.

Gamero, C. (2005). Análisis microeconómico de la satisfacción laboral. Madrid: Consejo Económico y Social.

García, M.A., Barbero, M.I., Ávila, I., \& García, M.C. (2003). La motivación laboral de los jóvenes en su primer empleo. Psicothema,15(1), 109-113.

Gazioglu, S., \& Tansel, A. (2006). Job satisfaction in Britain: individual and job related factors. Applied Economics, 38(10), 1163-1171. http://dx.doi.org/10.1080/00036840500392987

Ghazzawi, I. (2011). Does age matter in job satisfaction? The case of US information technology professionals. Journal of Organizational Culture, Communication and Conflict, 15(1), 25-54.

Goetz, K., Hasse, P., Campbell, S.M., Berger, S., Dörfer, C.E., Hahn, K. et al. (2015). Evaluation of job satisfaction and working atmosphere of dental nurses in Germany. Community dentistry and oral epidemiology, 44(1), 24-31. http://dx.doi.org/10.1111/cdoe.12186

Hofmans, J., De Gieter, S., \& Pepermans, R. (2013). Individual differences in the relationship between satisfaction with job rewards and job satisfaction. Journal of Vocational Behavior, 82(1), 1-9. http://dx.doi.org/10.1016/j.jvb.2012.06.007

Hoppock, R. (1935). Job Satisfaction. New York: Harperand Brothers.

Judge, T.A., Piccolo, R.F., Podsakoff, N.P., Shaw, J.C., \& Rich, B.L. (2010). The relationship between pay and job satisfaction: A meta-analysis of the literature. Journal of Vocational Behavior, 77(2), 157-167. http://dx.doi.org/10.1016/j.jvb.2010.04.002 
Judge, T.A., Thoresen, C.J., Bono, J.E., \& Patton, G.K. (2001). The job satisfaction-job performance relationship: A qualitative and quantitative review. Psychological Bulletin,127(3), 376-407. http://dx.doi.org/10.1037/0033-2909.127.3.376

Kooij, D.T.A.M., Jansen, P.G.W., Dikkers, J.S.E., \& De Lange, A.H. (2010). The influence of age on the associations between HR practices and both affective commitment and job satisfaction: A metaanalysis. Journal of Organizational Behavior, 31(8), 1111-1136. http://dx.doi.org/10.1002/job.666

Koustelios, A.D. (2001). Personal characteristics and job satisfaction of Greek teachers. International Journal of Educational Management,15(7), 354-358. http://dx.doi.org/10.1108/eum0000000005931

Lawler, E.E., \& Porter, L.W. (1967). The effect of performance on job satisfaction. Industrial Relations,7(1), 20-28. http://dx.doi.org/10.1111/j.1468-232x.1967.tb01060.x

Leigh, J.P. (1988). Assessing the importance of an independent variable in multiple-regression: Is stepwise unwise?. Journal of Clinical Epidemiology, 41(7), 669-677. http://dx.doi.org/10.1016/08954356(88)90119-9

Leung, J., Rioseco, P., \& Munro, P. (2015). Stress, satisfaction and burnout amongst Australian and New Zealand radiation oncologists. Journal of medical imaging and radiation oncology, 59(1), 115-124. http://dx.doi.org/10.1111/1754-9485.12217

Linz, S.J., \& Semykina, A. (2012). What makes workers happy? Anticipated rewards and job satisfaction. Industrial Relations: A Journal of Economy and Society, 51(4), 811-844. http://dx.doi.org/10.1111/j.1468232x.2012.00702.x

Linz, S., \& Semykina, A. (2013). Job satisfaction, expectations, and gender: Beyond the European Union. International Journal of Manpower, 34(6), 584-615. http://dx.doi.org/10.1108/IJM-06-2013-0149

Lloréns, F.J., \& Senise, M.E. (1996). Una evaluación económica del impacto salarial en las actitudes de los trabajadores ante el mercado laboral. Investigaciones Europeas de Dirección y Economía de la Empresa, 2(3), 13-25.

Markowits, Y., Boer, D., \& Van Dick, R. (2014). Economic crisis and the employee: The effects of economic crisis on employee job satisfaction, commitment, and self-regulation. European Management Journal, 32(3), 413-422. http://dx.doi.org/10.1016/j.emj.2013.09.005

Mehri, M., Iqbal, M.J., Hekmat, R.K., \& Ishaq, H.M. (2011). Impact of global financial crises on job security and job motivation of employees of banking sector in Iran. 2nd International Conference on Business and Economic Research. Malaysia (proceedings): Kedah. 
Mpeka, R.L. (2012). A study to examine the determinants of job satisfaction for professional accountants in Tanzania. Afro-Asian Journal of Finance and Accounting, 3(1), 15-33. http://dx.doi.org/10.1504/AAJFA.2012.046346

Norman, G.R., \& Streiner, D.L. (2008). Biostatistics: The Bare Essentials (3rd edition). Hamilton: McGrawHill Europe.

Okpara, J.O. (2004). Personal characteristics as predictors of job satisfaction: An exploratory study of IT managers in a developing economy. Information Technology and People,17(3), 327-338. http://dx.doi.org/10.1108/09593840410554247

Okpara, J.O. (2006). The impact of personal characteristics on the job satisfaction of public sector managers in a developing economy: Implications for personnel development. African Journal of Business and Economics Research, 1(1), 10-29.

Pérez, J.A. (1997). Motivación y satisfacción laboral: Retrospectiva sobre sus formas de análisis. Revista Española de Investigaciones Sociológicas, Reis 80, 133-167.

Petrescu, A.I., \& Simmons, R. (2008). Human resource management practices and workers job satisfaction. International Journal of Manpower, 29(7), 651-667. http://dx.doi.org/10.1108/01437720810908947

Platis, C., Reklitis, P., \& Zimeras, S. (2015). Relation between Job Satisfaction and Job Performance in Healthcare Services. Procedia-Social and Behavioral Sciences, 175, 480-487. http://dx.doi.org/10.1016/j.sbspro.2015.01.1226

Robles-García, M., Dierssen-Sotos, T., Martínez-Ochoa, E., Herrera-Carral, P., Díaz-Mendi, A.R., \& Llorca-Díaz, J. (2005). Variables relacionadas con la satisfacción laboral: Un estudio transversal a partir del modelo EFQM. Gaceta Sanitaria,19(2), 127-134. http://dx.doi.org/10.1157/13074368

Sánchez, S.M., Fuentes, F.., \& Artacho, C. (2007). La perspectiva de género en el análisis de la satisfacción laboral: una aplicación empírica mediante modelos logit y probit. Revista Cuadernos de Gestión,7(2), 55-67.

Sánchez-Sellero, M.C., Sánchez-Sellero, P., Cruz-González, M.M., \& Sánchez-Sellero, F.J. (2014). Características organizacionales de la satisfacción laboral en España. RAE-Revista de Administração de Empresas, 54(5), 537-547. http://dx.doi.org/10.1590/S0034-759020140507

Sánchez-Sellero, M.C., Sánchez-Sellero, P., Cruz-González, M.M., \& Sánchez-Sellero, F.J. (2016). Stability and Satisfaction at Work During the Spanish Economic Crisis. Prague Economic Papers, article first published online, 1-18. http://dx.doi.org/10.18267/j.pep.596 
Singh, P., \& Loncar, N. (2010). Pay satisfaction, job satisfaction and turnover intent. Relations Industrielles/Industrial Relations, 65(3), 470-490. http://dx.doi.org/10.7202/044892ar

Spagnoli, P., Caetano, A., \& Santos, S.C. (2012). Satisfaction with job aspects: Do patterns change over time?. Journal of Business Research, 65(5), 609-616. http://dx.doi.org/10.1016/j.jbusres.2011.02.048

Thompson, B. (1995). Stepwise Regression and Stepwise DiscriminantAnalysis Need Not Apply Here: A Guidelines Editorial. Educational and Psychological Measurement,55(4), 525-534. http://dx.doi.org/10.1177/0013164495055004001

Thompson, B. (2001). Significance, effect sizes, stepwise methods, and other issues: Strong arguments move the field. Journal of Experimental Education, 70(1), 80-93.

http://dx.doi.org/10.1080/00220970109599499

Tremblay, M., Chênevert, D., \& Hébert, A. (2012). Le rôle des conditions de travail dans la satisfaction et la loyauté des infirmières d'agence au Québec. Relations Industrielles/Industrial Relations, 67(3), 477-504. http://dx.doi.org/10.7202/1012540ar

Westover, J.H., \& Taylor, J. (2010). International differences in job satisfaction: The effects of public service motivation, rewards and work relations. International Journal of Productivity and Performance Management, 59(8), 811-828. http://dx.doi.org/10.1108/17410401011089481

Westover, J.H. (2012a). The job satisfaction-gender paradox revisited: A cross-national look at gender differences in job satisfaction, 1989-2005. Journal of Global Responsibility, 3(2), 263-277. http://dx.doi.org/10.1108/20412561211260557

Westover, J.H. (2012b). Comparative welfare state impacts on work quality and job satisfaction: A crossnational analysis. International Journal of Social Economics, 39(7), 503-525. http://dx.doi.org/10.1108/03068291211231687

Zhu, Y. (2013). A review of job satisfaction. Asian Social Science, 9(1), 293-298.

Intangible Capital, 2016 (www.intangiblecapital.org)

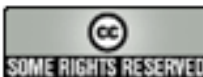

Article's contents are provided on an Attribution-Non Commercial 3.0 Creative commons license. Readers are allowed to copy, distribute and communicate article's contents, provided the author's and Intangible Capital's names are included. It must not be used for commercial purposes. To see the complete license contents, please visit http://creativecommons.org/licenses/by-nc/3.0/. 